\section{Kein erhöhtes Risiko für rheumatoide Arthritis durch antihormonelle Brustkrebs-Therapie}

Wadström $\mathrm{H}$ et al. Risk of breast cancer before and after rheumatoid arthritis, and the impact of hormonal factors. Annals of the Rheumatic Diseases 2020; 79: 581-586

Brustkrebs-Patientinnen haben kein erhöhtes Risiko, an Rheumatoider Arthritis (RA) zu erkranken. Zu diesem Schluss kommt jetzt eine schwedische Kohortenstudie. Die antihormonelle Therapie mit Tamoxifen oder Aromatasehemmern, die für die Behandlung eines hormonsensitiven Mammakarzinoms eingesetzt wird, stand im Verdacht, das Risiko für RA zu erhöhen. Dieser Zusammenhang konnte durch die Studie widerlegt werden.

Patientinnen äußern immer wieder die Sorge, dass Medikamente gegen Rheuma die Entstehung von Brustkrebs begünstigen könnten. Bei einer entzündlichen Autoimmunerkrankung wie der rheumatoiden Arthritis (RA) greift das Immunsystem körpereigene Strukturen an. Durch die Einnahme von Immunsuppressiva soll dies verhindert werden, denn diese unterdrücken Teile der körpereigenen Immunabwehr. Ein potenzieller Nachteil: Die Schwächung der Abwehr des eigenen Körpers macht ihn auch anfälliger für aufkommende Krebszellen. Frühere Studien berichten daher von einem erhöhten Krebsrisiko bei rheumatischen Erkrankungen.

Dies gilt aber nicht für alle Krebsarten: „Interessanterweise zeigen vergangene Studien ein reduziertes Brustkrebsrisiko bei Frauen mit RA“, erklärt EULAR-Kongresspräsident Prof. Iain B. McInnes aus Glasgow, Schottland. Frühere Studien haben gezeigt, dass die antihormonelle Therapie mit Tamoxifen oder Aromatasehemmern, die bei Frauen mit Brustkrebs eingesetzt wird, mit einem häufigeren Auftreten von Gelenkschmerzen einhergeht. Ob sie jedoch auch zu einer eindeutigen RA führen können, blieb umstritten.

In einer bundesweiten schwedischen Kohorten-Studie sind Wissenschaftler nun der Frage nachgegangen, ob es einen Zusammenhang zwischen Brustkrebs, einer antihormonalen Therapie und RA gibt. Hjalmar Wadström, Doktorand am Karolinska Institutet Stockholm, hat dazu 15921 Frauen identifiziert, bei denen zwischen 2006 und 2016 RA diagnostiziert wurde. Die Daten wurden mit Brustkrebsrisikofaktoren in Verbindung gebracht und das Risiko von Brustkrebs bei Frauen mit RA und das RA-Risiko bei Frauen mit Brustkrebs wurde untersucht. Diese wurden dann mit einer von Alter und Geschlecht gleich strukturierten Kontrollgruppe verglichen. Das Ergebnis: Das Brustkrebsrisiko für Frauen mit RA war geringer als für Frauen mit gesunden Gelenken. Außerdem wurde kein Zusammenhang zwischen einer antihormonalen Brustkrebsprophylaxe und späterer RA gefunden.

„Unsere Untersuchungen zeigen, dass das Brustkrebsrisiko bei RA zwar verringert ist, dieser Zusammenhang jedoch nicht ohne weiteres durch herkömmliche Risikofaktoren für Brustkrebs erklärt werden kann“, fasst Wadström die Ergebnisse der Kohortenstudie zusammen. „Auch der Einsatz einer antihormonellen Therapie bei Brustkrebs scheint das Risiko für eine RA nicht zu erhöhen“, ergänzt Wadström und schlussfolgert mit Bezug auf frühere Studien, dass die RA-Erkrankung in der klinischen Praxis wahrscheinlich nur einen begrenzten Einfluss auf die Krebsentwicklung gehabt hat.

„Wir wissen jetzt, dass Brustkrebs bei Patientinnen mit RA seltener vorkommt und dass Tamoxifen- und Aromatasehemmer im Hinblick auf das Risiko einer zukünftigen RA zumindest in dem ersten beobachteten Zeitraum sicher zu sein scheinen - das sind wichtige Informationen für die Patientenberatung“, erklärt EULAR Scientific Chair Prof. John Isaacs von der Newcastle University, UK. „Trotzdem sollten sich alle Patienten mit einer symptomatischen Rheuma-Erkrankung regelmäßig einem ihrem Alter angemessenen Tumorscreening unterziehen “.

Nach einer Pressemitteilung des Europäischen Rheumatologenkongresses 